\title{
Nicht-Sinn und die Konstitution des Sozialen
}

ALBRECHT KOSCHORKE

I.

Den Anstoß für die folgenden Überlegungen bildet ein Satz von Bernd Giesen, der auf knappem Raum, ebenso lapidar wie selbstbewusst, eine Definition von >Kultur< leisten will:

»Wir verstehen unter Kultur also jenen sinnstiftenden Horizont, der im Augenblick des Handelns für Ego und Alter svorausgesetzt c wird, und der damit die Unterwelt des Absurden latent hält« (Giesen 2004: 76).

Sieht man von der »Unterwelt «, dem »Absurden« und der »Latenz« vorerst ab, dann befindet sich diese Äußerung in bester soziologischer Nachbarschaft. Schon Max Weber hatte ja die Soziologie auf das »Verstehen« verpflichtet und ihr Grundelement, das »Handeln«, als wesentlich »sinnhaft« bestimmt. Berühmt sind die axiomatischen Eingangssätze von $>$ Wirtschaft und Gesellschaft $\iota$, in denen Sinn- und Sozialdimension miteinander verschränkt werden:

mHandeln soll [...] ein menschliches Verhalten (einerlei ob äußeres oder innerliches Tun, Unterlassen oder Dulden) heißen, wenn und insofern als der oder die Handelnden mit ihm einen subjektiven Sinn verbinden. >Soziales Handeln aber soll ein solches Handeln heißen, welches seinem von dem oder den Handelnden gemeinten Sinn nach auf das Verhalten anderer bezogen wird und daran in seinem Ablauf orientiert ist« (Weber 1980: 1).

Daran knüpfen Weber und seine späteren Exegeten zwar eine Reihe von Problematisierungen an - etwa ob der sinnhafte Charakter menschlichen Handelns erschöpfend mit Begriffen wie Intentionalität und Zweckrationa- 
Grundvoraussetzung nicht, dass wir uns als soziale Wesen in Sinnhorizonten bewegen, ja dass es eine Dauerpräsenz von ıSinn ‘ im sozialen Raum gibt. Soweit ich sehe, bleibt die Soziologie des 20. Jahrhunderts in ihren Hauptrichtungen diesem hermeneutischen Vermächtnis treu. Für Luhmann, der mit der Kategorie `Sinn< ein an sich fremdes Element phänomenologischen Ursprungs in die Systemtheorie implantiert, ist Sinn eine »evolutionäre Errungenschaft«, die >Erleben $<$ und $>$ Handeln $<$ in den Horizont überschüssiger Verweisungen stellt. Luhmann spricht sogar von einem »Sinnzwang, der allen Prozessen psychischer und sozialer Systeme auferlegt ist« (1988: 92). Denn Sinn ist für ihn

»eine unnegierbare, differenzlose Kategorie. Ihre Aufhebung wäre im strengsten Sinne rannihilatio - und das wäre Sache einer undenkbaren externen Instanz. >Sinnlosigkeit< kann deshalb nie durch Negation von Sinnhaftigkeit gewonnen werden. Sinnlosigkeit ist ein Spezialphänomen, es ist überhaupt nur im Bereich der Zeichen möglich und besteht in einer Verwirrung von Zeichen. Ein Durcheinanderbringen von Objekten ist niemals sinnlos, ein Trümmerhaufen zum Beispiel ist sofort als solcher erkennbar, und zumeist sieht man auch gleich mit, ob er auf Alter oder Erdbeben oder >Feindeinwirkung` zurïckzuführen ist« (ebd., 96).

Mit einem definitorischen Handstreich macht sich die Soziologie im Nachkriegsdeutschland nicht nur von allen existenzialistischen oder gar nihilistischen Versuchungen frei, sondern entsorgt auch gleich noch die Erinnerung an den Krieg - schließlich sind auch durch Feindeinwirkung entstandene Trümmerhaufen >erkennbar und insoweit keine schlechthin sinnlosen Phänomene. >Sinn< ist selbstreferenziell und zirkulär in sich verschlossen, deshalb kann sein Geltungsbereich weder verneint noch verlassen werden. >Erfahrung der Sinnlosigkeit stellt für Luhmann keinen soziologischen Tatbestand dar (ebd., 109).

So kithl sich die Diktion in diesem Fall gibt, das Beharren auf der sozialen Omnipräsenz von Sinn (oder umgekehrt, die Marginalisierung seines Fehlens) erinnert an theologische Denkfiguren. Das wird deutlicher in einer anderen Spielart des soziologischen Konstruktivismus, nämlich bei Peter Berger und Thomas Luckmann. Auch die Alltagswelt, um die es Berger und Luckmann zu tun ist, bildet keinen sinnfreien Raum. Sie ist in >Sinnprovinzen $<$ zergliedert und in verschiedenen »Ebene[n] der Sinnhaftigkeit《 (Berger/Luckmann 2004: 87) übereinandergeschichtet. Diese Schichten oder Parzellen wiederum werden in »symbolischen Sinnwelten« zusammengeführt. »Wir meinen damit«, schreiben Berger und Luckmann, »synoptische Traditionsgesamtheiten, die verschiedene Sinnprovinzen integrieren und die institutionale Ordnung als symbolische Totalität überhöhen $[. ..] \ll($ ebd., 102). - »Die symbolische Sinnwelt ist als die Matrix aller gesellschaftlich objektivierten und subjektiv wirklichen Sinnhaftigkeit zu verstehen« (ebd., 103). Wie in einer Fußnote vermerkt wird, ist dieser Begriff dem Religionsbegriff bei Durkheim verwandt (ebd, 102). Ohne eine solche Sinnwelt, die alles überwölbt und integriert, würde unsere Alltagswelt auseinanderfallen. Dazu nur zwei Zitate:

»Sinnprovinzen, die ohne eine >oberste symbolische Sinnwelt unbegreifliche Enklaven in der Wirklichkeit der Alltagswelt bleiben müßten, gruppieren sich mit ihrer Hilfe zu einer Hierarchie der Wirklichkeiten, die dadurch begreiflicher und weniger erschreckend wirkt« (ebd., 105).

Und: Sinnwelten »sind wie schützende Dächer über der institutionalen Ordnung und über dem Einzelleben« (ebd, 109).

Diese wenigen Belegstellen sollten hinreichend sein, um zu zeigen, dass in Berger/Luckmanns Alltagssoziologie eine ganze Menge Metaphysik Zuflucht findet. Denn die Autoren errichten ja einen die Gesamtheit der sozialen Welt durchgreifenden Instanzenzug: Da gibt es individuelle Sinnentwürfe; große und kleine, obskure und helle, periphere und zentrale »Sinnprovinzen«; es gibt Institutionalisierungen jeder Art, die auf Legitimation und damit sozusagen wiederum auf >Sinnbarmachung schließlich symbolische Sinnwelten, die über all dem ein schützendes Dach bilden. Und wenn das Dach einstürzt, so legt die Darstellung nahe, zerfallen auch die darunter liegenden Stockwerke des Gebäudes.

So wird die Soziologie zu einer Wissenschaft von den letzten Fragen:

$» \mathrm{Da}$ der Tod [...] die ärgste Bedrohung für die Gewißheit der Wirklichkeiten des Alltagslebens darstellt, braucht nicht eigens betont zu werden. Die Integration des Todes in die oberste Wirklichkeit des gesellschaftlichen Daseins ist deshalb für jede institutionale Ordnung von größter Wichtigkeit. Demzufolge ist die Legitimation des Todes eine der wichtigsten Funktionen symbolischer Sinnwelten« (ebd., 108).

II.

Als Literaturwissenschaftler hat es mich immer gewundert, wie unbefangen die Nachbardisziplin Soziologie Prozesse der Verständigung zwischen sozialen Akteuren, der Sinnbezogenheit von Ego-Alter-Interaktionen, der gesellschaftlichen Integration durch Sinn und schließlich der erfolgreichen Legitimation auf allen Ebenen zu ihrer Grundlage macht. Natürlich werden dabei ganz unterschiedliche Sinnkonzepte verwendet, die vom Formalismus bloßer kommunikativer Anschlussermöglichung bei Luhmann bis zu Berger/Luckmanns symbolischen Sinnwelten reichen, die uns vom Gedanken des Todes abschirmen. Auch wird nicht immer konsistent zwi-

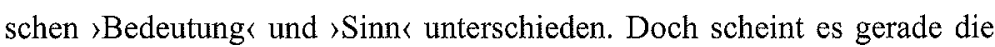


suggestive Unschärfe des Sinnbegriffs zu erlauben, sogar - wie in dem eingangs zitierten Satz von Bernd Giesen - die Kultur als ganze zu einem »sinnstiftenden Horizont« zu erklären.

Aus literaturwissenschaftlicher Sicht ist das erstaunlich. Schließlich liegt die Gründungsphase der Soziologie in einer Zeit, die literarisch von der Sprachkrise der Moderne geprägt war: die gesamte Dichtung des 20 . Jahrhunderts ist eher mit der Absenz, ja mit der Zerstörung von Sinnbezügen als mit Sinnstiftung befasst; die Literaturtheorie seit den siebziger Jahren hat das durch eine tiefgreifende Infragestellung hermeneutischer $\mathrm{Ge}$ wissheiten nachvollzogen. Während den Dichtern der Klassischen Moderne die Worte bekanntlich wie Pilze im Munde vermodern, entsteht >nebenan< eine Disziplin, die >Gesellschaft‘ als eine Sphäre gelingender Verständigung ansieht; während Dada, absurdes Theater, hermetische Lyrik und eine sich der Beschreibbarkeit der Welt entschlagende Prosa Inkommunikabilität zu ihrem Produktionsprinzip erheben, fühlen sich die Sozialtheoretiker nicht nur dem Funktionieren, sondern auch der legitimatorischen Kraft kommunikativer Prozesse verpflichtet. Bestenfalls räumen sie der Verweigerung von Sinn einen Ort in den Katakomben des kulturellen Bewusstseins ein, wo es dann von Zeit zu Zeit aus der Latenz hervorbricht und herrschende semantische Konventionen erschüttert.

Es scheint mir aber fraglich, ob kulturelle Vorgänge mit einer solchen Dialektik zwischen sinnhafter Normalität und ihren flankierenden Latenzschutzmaßnahmen einerseits, Sinnzusammenbrüchen andererseits ${ }^{1}$ hinreichend erfasst werden können. Stattdessen möchte ich dafür plädieren, von einem grundlegenderen (und skeptischeren) Kulturbegriff auszugehen und gewissermaßen den Fokus der Kamera tiefer zu stellen: auf jene noch vorbegriffliche und präsemantische Zone, in der die Entscheidung, was als signifikant (und damit >sinnfähig`) und was als insignifikant gilt, noch gar nicht getroffen ist, in der die dazu nötigen Regimes der Bedeutungsproduktion und -fixierung sich allererst ausbilden und verfestigen - was auch heißt, dass noch keine institutionellen Vorkehrungen eine >Unterwelt< des >Absurden<, >Dämonischen oder des >Traumas von der Tageswelt der ihres Sinnes gewissen Handlungen absondern. Hierzu findet man übrigens reiche Hinweise in den Überlegungen, die Bernd Giesen (2004) in dem erwähnten Aufsatz zur Produktion von sozialer Latenz anstellt.

Es ist mir also in keiner Weise darum zu tun, der Rede von Gesellschaft oder Kultur als Sinnsphäre ein nihilistisches Credo entgegenzusetzen oder die Soziologie zu >dekonstruieren mehr darin, >Sinn als einen unter bestimmten Bedingungen erzeugten $E f$ fekt und nicht als schlechthinnige Prämisse kultureller Aktivität zu behandeln. Deshalb möchte ich im Folgenden aus kultursemiotischer Perspektive einige Beobachtungen sammeln, die vor die Alternative zwischen so-

1 So die Argumentationslinie bei Bernhard Giesen (2004). zialem Sinn und Nicht-Sinn zurückgehen. >Nicht-Sinn ist hierbei keinesfalls mit Sinnlosigkeit gleichzusetzen. Ob etwas sinnvoll oder sinnlos ist, ist eine Frage der Befindlichkeit oder der Weltanschauung, das heißt eine schon innersemantische Frage. Ob, wann, wo und mit welcher Intensität Sinnzusammenhänge auf- oder abgebaut werden, ist demgegenüber eine Frage, die auf die strukturellen, oder noch genauer: infrastrukturellen Dispositionen kulturellen Zeichengebrauchs zielt. Auch die Ausdünnung oder Zersetzung von Sinn ist ein gesellschaftlich relevanter Vorgang - nicht weil man, wie im Fall avantgardistischer Kunstwerke häufig geschehen, Akte der semantischen Obstruktion mit dialektischer Raffinesse nachweisen kann, dass sie doch irgendeinen kulturellen Sinn beanspruchen, sondern weil sie eine Stelle im Kontinuum zwischen Fülle und Absenz von Sinn besetzen, dessen Gesamtheit die Kultur bildet. Und beide Vorgänge, Sinnzuschreibung und Sinnentzug, spielen sich im hellen Licht des Tages ab.

Ein solches Untersuchungsdesign hängt von verschiedenen Prämissen ab, die ich hier nicht im Einzelnen diskutieren kann. Dazu gehören: die Ungleichmäßigkeit kultureller Topographien, die Unvollständigkeit und begrenzte Reichweite von hegemonialen Semantiken, die der Welt ihren Sinn aufprägen wollen; die Arbeit, die zu ihrem Unterhalt aufgewendet werden muss; die Zeit, die dieser Unterhalt kostet; die Instanzen, die dazu erforderlich sind; und nicht zuletzt die wechselseitige Durchdringung, Überlagerung, Annullierung konkurrierender Semantiken im sozialen Feld.

\section{III.}

Ich würde nämlich geltend machen, dass gerade alltagsweltlich die Referenz auf ১Sinn « nur in seltenen Fällen aktiviert wird. Man muss allerdings zunächst Ebenen von Sinnbildung unterscheiden. Zwar gibt es in alltäglicher Kommunikation so etwas wie eine basale Sinnerwartung (z.B. dass der andere auf meine kommunikativen Offerten nach erwartbaren Mustern reagiert oder dass gesprochene/geschriebene Sätze auf elementare Weise verständlich sind). Aber gerade weil diese Erwartungen normalerweise erfüllt werden oder ihre Nicht-Erfüllung alltagsweltlich marginalisiert werden kann, wird >Sinn ‘ in einem anderen als technischen Sinn selten explizit zum Problem. Gewöhnlich stellt sich die `Sinnfrage〈 nicht, so wenig wie sich in $99 \%$ aller Lebenslagen die Frage nach $>$ Identität ( (eigener oder fremder) stellt. Es müssen sehr viele Irritationen zusammentreffen, bevor die Referenz auf > Sinn 〈 ins Zentrum der Aufmerksamkeit rückt.

Man könnte also geradezu ein Axiom der 'Sinnvergessenheit< des alltagsweltlichen Handelns aufstellen. Die meisten Alltagsfunktionen sind gegenüber der Alternative von sinngemäßem Verstehen oder Nichtverste- 
hen in hohem $\mathrm{Ma} 3$ indifferent oder tolerant. Und zwar deshalb, weil nur ein Bruchteil der sozialen Funktionen von solchen Verstehensprozessen abhängt. Apparate, Verfahren, Einrichtungen, die unser alltägliches Funktionieren ermöglichen, sind nicht primär sinnhaft; >Sinn bildet ihnen gegenüber oft nur eine nachträgliche Attribution.

Dies gilt umso mehr, je höhere Integrationsebenen von Sinn im Spiel sind. Eine Gesellschaft, die jedes ihrer Elemente mit der Frage nach Sinn und Legitimität konfrontierte, würde bald eine Art von >Sinninfarkt ‘ erleiden. Es mag berufsmäßige Legitimatoren geben, die ihr diese Frage als Dauerproblem aufzwingen wollen. Das hat aber eher einen negativen als einen positiven Grund. >Sinn ( (auf höherer Ebene) ist keine Voraussetzung dafür, zu leben - und gut zu leben. Lust, Genuss, organisches Wohlgefühl, Liebe, Befriedigung von Trieben oder das Begehren danach haben keine ursprüngliche Referenz auf $>$ Sinn $<$. Die Sinnfrage drängt sich meist erst in der Form einer Perhorreszierung von Sinnlosigkeit auf: das heißt als ein privatives Phantasma, ein Phantasma des Mangels. Es gibt bestimmte Berufsgruppen, die von diesem Phantasma besonders heimgesucht werden oder mit ihm ein besonderes Interesse verbinden. Das sind die Legitimationsexperten - Priester, Philosophen, Ideologen, Angehörige von Deutungseliten aller Art. Diese Experten wollen eine Dauerreflexion auf >Sinn institutionalisieren. Ihnen ist der Gedanke einer A-Semantik, einer Sinn- und Bedeutungsindifferenz des Lebens ein Gräuel, und deshalb müssen sie ihm entgegenarbeiten.

Dabei dient ihnen der Tod als machtvollstes Argument. Aber eine ständige Bezugnahme auf den Tod als End- und Zielpunkt des menschlichen Lebens würde, nüchtern gesprochen, alle Alltagsfunktionen blockieren. Sie ist infolgedessen auch nur außeralltäglich realisierbar: in Eremitagen und Klöstern, im >memento mori< von barocken Gedichten, christlichen Predigten oder Gedenkveranstaltungen modernen Typs. Deshalb sind religiöse oder weltliche Tröstungen über den Tod - anders, als sie vorgeben - ihrem Wesen nach flüchtiger und provisorischer Natur. Sie reagieren auf Momente von Sinnkrisen, etwa bei Todesfällen. In den (lebensnotwendigen!) Anforderungen und Zerstreuungen des Alltags gravitieren sie nach dem Krisenereignis, das zu ihrer rituellen Aktivierung geführt hat, bald wieder an ihren marginalen Platz zurück. Und das ist nicht, wie die Kulturkritik will, erst der Effekt einer nihilistischen Moderne, sondern >war schon immer sor. Wer das Leben nur sub specie aeternitatis betrachtet, kann nichts wichtig nehmen, kann nicht lieben, seine Feldarbeit nicht tun, seine Kinder nicht nähren, seinen Beruf nicht ausfüllen; er steht in der Gefahr, lebensunfähig zu werden.

Was die Menschen vor dem Nichts abschirmt, sind also nicht die >Sinnwelten als »schützende Dächer über der institutionalen Ordnung und über dem Einzelleben«, wie Berger/Luckmann (2004: 109) meinen. Das wäre aus der verengten Perspektive von Legitimationsexperten gedacht, die ,Nicht-Sinn mit Sinnlosigkeit gleichsetzen, die also alles, was sie nicht erfolgreich bearbeiten, als Vakuum und Absenz erscheinen lassen, wodurch sie sich - gleichsam metaphysisch - unentbehrlich machen. Wie die Soziologie weiß, ist Legitimierung eine soziale Aktivität, die von bestimmten Spezialisten ausgeübt und zur Perfektion gebracht werden kann. Diese Spezialisten bringen in ihr Tun Perspektiven und Interessen ein, die nicht ohne weiteres verallgemeinerbar sind; ihr Geschäft ist also nicht das Geschäft der Soziologen².

Die professionellen Deutungseliten setzen nämlich einen Systemzwang in Gang, der sie als Berufsgruppe unentbehrlich zu machen scheint - einen Zwang der Ebenenhierarchie, weil ja kleinräumige Sinnbezirke umgreifenden Sinnprovinzen und diese einer ihrem Selbstverständnis nach totalen Sinnwelt eingefügt oder untergeordnet werden müssen, um sich gegen den omnipräsenten Nicht-Sinn an ihren Rändern gleichsam abzudichten. Die Konstruktion einer solchen Ebenenhierarchie bedarf einer großen systematischen Anstrengung; sie zwingt zu theoretischen Abstraktionen, die das Vorhandensein einer dazu fähigen Profession notwendig macht und rechtfertigt. Denn solche Systeme sind empfindlich gegenüber Unvollständigkeiten, die sie gedanklich zum Einsturz bringen. Insofern ist das ganze Unternehmen von einem inneren shorror vacui<, einem Horror gegenüber dem Nicht-Stellen der Sinnfrage, getrieben.

Umfassende Rechtfertigung in dem hier beschriebenen Sinn wäre nur unter hochspezialisierten, dem Alltag entzogenen und entsprechend unwahrscheinlichen Bedingungen zu erzielen. Solche Bedingungen sind im Normalfunktionieren von Gesellschaften nicht herzustellen. Pragmatisch würde die ständige Referenz auf Sinn, d.h. permanenter `Sinnstress〈, zum Zusammenbruch der alltäglichen Funktionen und Verrichtungen führen. Würde eine Gruppe von Legitimatoren tatsächlich alle Vorgänge kontrollieren, so würde dies wohl nur um den Preis einer geradezu terroristischen Reinigung der sozialen Realitäten gelingen. Davor schützt normalerweise, dass Systemzwänge jeweils nur eine begrenzte interne und äußere Reichweite haben und dass die Energie, die erforderlich ist, um sie aufrechtzuerhalten, nicht ständig in gleichem Maß aufgebracht werden kann. Man

2 Wie es sich anhört, wenn man beides - die Metaphysik besorgter Deutungsexperten und die soziologische Analyse - miteinander verwechselt, kann man in einschlägigen Fachlexika studieren. Eine Stilprobe: »Mit der Herausbildung $u$. Entfaltung der mod. Ges, sind überkommene soziokult. Wertsysteme relativiert und erschüttert worden, haben sich Traditionen abgeschwächt oder aufgelöst, haben viele Kulturelemente u. >soz. Tatsachen ihren Selbstverständlichkeitscharakter eingebüßt. Die allg. Sinnorientierung ist dadurch entstabilisiert $u$. verunsichert worden. Für absolut gültig gehaltene Wert- u. Sinnvorstellungen unterliegen der Erosion durch krit.-rationale Reflexion. Sinnverluste erschweren das soz. Zus.leben $u$. führen zu psych. Problemen (Depression, neurot. Störungen)« (Hillmann 1994: 783). 
hat es also, der Struktur menschlicher Aufmerksamkeit gemäß, eher mit wandernden und in ihrer Extension ständig schwankenden 'Sinnfenstern als mit geschlossenen, totalistischen Sinngebäuden zu tun.

Das wirft die Frage auf, was denn die Menschen vor dem Nichts abschirmt, wenn es nicht ihre hochelaborierten und fugendichten Sinnwelten sind. Die Antwort darauf kann mit den Mitteln einer kultursemiotischen Reichweitenanalyse, in diesem Fall in Hinsicht auf die begrenzte Reichweite des Sinn-Begriffs selbst, gegeben werden. Sie setzt die Anerkennung der Tatsache voraus, dass die Sinnfrage situativ eben nicht weit über den Gottesdienst oder das Beerdigungszeremoniell oder die Lektüre eines erschütternden Buches hinausreicht. Und auch dort, wo diese Frage im Zusammenhang einer Lebenskrise durchlitten wird, kreuzt und vermengt sich die Aufmerksamkeit auf >Sinn く bzw. >Sinnlosigkeitく ständig mit anderen Aufmerksamkeiten. Das mag man für ein Defizit halten - oft pejorativ dem Alltag, oberflächlichen Nebenmenschen, der Zerstreuung durch Geselligkeit und Massenmedien zur Last gelegt -, aber es bietet am Ende einen effektiveren Schutz vor dem >Einbruch des Nicht` als jedes noch so elaborierte System der Rechtfertigung.

\section{IV.}

Man kann diese Überlegungen durch ein Gedankenexperiment illustrieren, nämlich die Beobachtung einer kollektiven sakralen Handlung. In deren Mitte wird man ein rituell stark reglementiertes, von einem hohen Artikulationsniveau geprägtes, theologisch äußerst durchdachtes und sinnbeladenes Geschehen erwarten. In der heiligen Verrichtung trägt sich ja die Begegnung der Gemeinde mit dem Göttlichen zu; oder es wird stellvertretend Fürsprache für die Gemeinde gehalten, was eine Berufsrolle und professionelle Ausbildung der Protagonisten des Rituals sowie Kaskaden der Abgrenzung (entlang der Leitunterscheidung heilig/profan) notwendig macht. Die Mitte des Ritus wird in der Regel von einem inneren Kreis privilegierter Gläubiger umgeben, die mit der gepflegten Semantik des Sakralen vertraut sind und sich entsprechend verhalten (mitsprechen, mitsingen, mittanzen, oder auch das Tun der Priester fachmännisch observieren). Zur Peripherie der sakralen Handlung hin nimmt der Grad von >semantischer Kontraktion< und Artikuliertheit ab. Die Aufmerksamkeit teilt sich; das Verständnis für die Details der heiligen Handlung stößt an Grenzen der Einsicht, des Respekts und der Bereitwilligkeit; die Unruhe des Profanen mischt sich hinein, mit all den typischen Zersetzungshandlungen am Rand von Hochsemantiken: Geschwätz, Kichern, spöttische Bemerkungen, Langeweile, Schlaf, notorische Unrast, Kommen und Gehen. Alles in allem herrscht hier eine Tendenz zur sozialen >Verflüssigung ` vor, in wachsen- dem Kontrast zu dem statuarischen Verhalten, das im Zentrum des rituellen Vorgangs geboten ist.

Semantisch geht die Unruhe am Rand der heiligen Handlung mit einer zunehmenden Desartikulation und Bedeutungsauflösung einher. In gewisser Weise verhält sich die Peripherie also konträr zum sakralen Geschehen, aber nicht durch eine zielgerichtete Gegenbewegung, sondern durch Entzug von strukturierender Energie, Senkung des Komplexitätsniveaus, Entropie, Rauschen. Gleichwohl bleibt die Peripherie parasitär und widersprüchlich auf das Geschehen im Zentrum bezogen, indem sie dessen Attraktion abwehrt und so einen gleichsam mittleren Aggregatzustand aufrechterhält. Die Abwehr stellt einen Reflex auf den hegemonialen Anspruch des Zentrums der Sinnsphäre dar; sie kann mit - in der Regel kurzlebiger - artikulierter Subversion einhergehen, etwa durch Witzereißen oder Spott über die beteiligten Würdenträger. Albernheit als Sinnzersetzung bildet insofern eine gegenstrebige Ergänzung zum Ernst des hochsemantischen Aktes.

Die Unruhe der Peripherie übt - wenn man sie nicht nur als Störung verurteilt (was die Betrachtungsweise vom Zentrum aus wäre) - eine mehrfache Wirkung aus. Erstens schränkt sie die Reichweite des Ernstes und der Feierlichkeit ein, mit der sich die sakrale Handlung Autorität spendet. Sie arbeitet also der Schöpfung und Allokation von Sinn bis zu einem gewissen Grad entgegen, lässt die artikulierte Semantik des zentralen Geschehens in Indifferenz und Nichtverstehen auslaufen. Aber dies ist nicht bloß als ein negativer Prozess anzusehen. Zweitens nämlich werden Sinnsysteme auf diesem Weg überhaupt erst von einer größeren Menge angeeignet und in einen kollektiven Bestand verwandelt. Hochartikulierte Semantiken bleiben zwangsläufig sozial exklusiv und können in dieser Form nur begrenzt Zusammengehörigkeit stiften. Sie müssen sozusagen >kleingearbeitet werden, um sich mit größerer Reichweite und Durchdringungstiefe vergesellschaften zu lassen. Zerstreuung von Sinn bildet also kein Hindernis für die Bildung größerer Gruppen, sondern arbeitet ihr zu. Auf Dauer wird kein Sinnsystem sozialen Erfolg haben, das nicht die Möglichkeit von Teilhabe auf unterschiedlichen Artikulations- und Energiestufen bereitstellt.

Drittens schließlich entstehen im Gefälle der Dissemination Spielräume der Abweichung und des Ungehorsams, aus denen neue Zentren der Artikulation, mit anderen Worten: Gegensemantiken, erwachsen können. Allerdings geschieht dies nur unter bestimmten Bedingungen. Denn vermutlich sind an solchen Gegenbildungen fast immer Abzweigungen der alten zentralen Eliten oder neu entstandene Expertokratien beteiligt. Das Verhältnis von Gegensemantiken zum herrschenden Sinnsystem ist ja seinerseits artikuliert, nicht dissipativ. Um die aus der Marginalität heraus entstehenden Gegenzentren lagern sich zudem die gleichen Ringe von Desartikulation und Sinnverminderung an - jedenfalls ab einem bestimm- 
ten Grad von Kodifizierung und institutioneller Festigung - wie um die alten hegemonialen Gebilde.

Daraus lässt sich der Schluss ziehen, dass man grundsätzlich zwischen >kalter (durch Effekte der Entropie) und sheißer Peripherie (durch Inversion des semiotischen Feldes und Ausbildung neuer Zentren der Sinnverdichtung) unterscheiden muss, auch wenn sich in der gesellschaftlichen Empirie beide ineinander vermischen mögen. Wie dem auch sei, das Beispiel sollte verdeutlicht haben, dass die Allokation von Sinn im sozialen Raum als Variable und nicht als Konstante aufgefasst werden muss.

Es gibt nämlich, das würde ich behaupten, sowohl in synchroner als auch in diachroner Perspektive wechselnde 'Sinndichten Reichweiten von sinnhafter Integration. In diachroner Perspektive: Sinnhaftes kann als solches rabsterben<, sich verdinglichen und seine originäre Bedeutung verlieren, um dann mit neuem Sinn saufgeladen rum der kulturellen Semiosis zurückgeführt zu werden. Alle kulturellen Dinge haben solche Sedimentschichten; oft hängt es vom situativen Kontext ab, ob sie - oder bestimmte Teile bzw. Aspekte von ihnen - zu >Sinn belebt werden oder stumm bleiben. Die $»$ Reichweite der sinnhaften Integration«, von der Berger/Luckmann (2004: 102f.) gelegentlich sprechen, ist auch in Hinsicht auf die historische beziehungsweise archäologische Dimension veränderlich.

Allein seiner Bauart nach ist Sinn, und gerade komplexer Sinn, in hohem Maß synkretistisch. ${ }^{3}$, Neuer Sinn ist also aus >altem Sinn gemacht und verwendet Bauteile davon. >Neuer Sinn $<$ kann sich aber auch um verlorenen, vergessenen oder sogar insistenten Nicht-Sinn herum konfigurieren. Ein Beispiel für ersteres sind Kultstätten, an denen sich ganz unterschiedliche, einander fremde Religionen abfolgen. Dabei werden oft nicht mehr verstandene Symbole oder Dinge in neue kultische Sinngebungen inseriert. Ein Beispiel für das zweite ist die Persistenz von Eigennamen in anderssprachlichen Zusammenhängen oder von offenkundig nicht sinnhaften Lexemen. Diese werden häufig zu >Rätselwörtern und ziehen eine besondere semantische Energie auf sich - sei es, dass man sie etymologisch der eigenen Sinnwelt anzuverwandeln versucht, sei es, dass sie als eine Art magischer Fremdkörper zum Kristallisationskern von narrativen Kontexterzeugungen werden

3 »Konzeptionen, die symbolische Sinnwelten stützen, führen immer zur Systematisierung kognitiver und normativer Legitimationen, die in naiver Form schon längst in der Gesellschaft vorhanden waren und in ihrer symbolischen Sinnwelt in kristallisierter Form erscheinen. Mit anderen Worten: das Material, aus dem die Stützkonzeptionen bestehen, ist meistens nichts anderes als eine Weiterverarbeitung der Legitimationen der einzelnen Institutionen auf der höheren Ebene theoretischer Integration« (Berger/Luckmann: 117).
Man könnte daraus eine anthropologische These ableiten, nämlich dass Menschen Nicht-Sinn nicht >ertragen< und ihn sich mit allen Mitteln anzuverwandeln versuchen - so wie man sich kulturell Fremdes aneignet und einverleibt. Aber das hieße, solche Vorgänge zu einseitig auf ein UrBedürfnis nach Sinn hin zuzurichten. Es gibt ja auch den umgekehrten Prozess, nämlich den Abbau besonders von hohem oder sakralem Sinn durch Entsemantisierung, Entstellung, Zerstreuung, Veralberung. So hat sich bekanntlich aus den Abendmahlsworten »Hoc est corpus meum«, also aus der zentralen semantischen Transformationsformel des Christentums, das sinnlose Zauberwort `Hokuspokus gebildet, um nur ein augenfälliges Beispiel zu nennen. Und beides, das >corpus mysticumı und dessen dämonisierende Spottformel, gehört auf gleiche Weise zum Raum der christlichen Kultur. Kulturelle Semiosis bewegt sich innerhalb der gesamten Bandbreite zwischen Semantisierung und Entsemantisierung. Unterscheidungen des Typs Tag/Nacht, hoch/tief, offiziell/subversiv, manifest/latent, sinnvoll/sinnlos sind ihrerseits nichts anderes als semiotische Operationen, und zwar solche, die im Einzugsbereich von semantischen Hegemonien getroffen werden.

Man muss überdies in Rechnung stellen, dass jede Gesellschaft in synchroner Perspektive aus vielen unterschiedlichen Sinnsphären besteht, die sich überlagern, verstärken, ignorieren, wechselseitig entkräften oder verfremden - bis hin zu offen konflikthaften Antagonismen, jedenfalls auf bestimmten kommunikativen Frequenzen. Selbst hochintegrierte hierarchische Sinnsysteme, die sich selbst den Status der Überlegenheit zuweisen, sind unter diesem Aspekt zunächst einmal nur ein `Sprachspiek (im Wittgensteinschen Sinn) unter anderen Sprachspielen - auch wenn es von diskursiv überlegenen Sinnexperten gespielt wird (wie z.B. das mittelalterliche Kloster in einer volksreligiösen, bäurischen Umgebung). Auch wo eine Sinnwelt universelle Geltung beansprucht, bleibt sie eine unter mehreren konkurrierenden Sinnwelten mit ihrem eigenen partikularistischen Hintergrund, etwa in Hinsicht auf die Besonderheit ihrer Trägerschicht.

\section{V.}

Wenn wir ein derart komplexes und variationsfähiges Modell konstruieren, wird es schnell als Irrtum erkennbar, dass Glaubenssysteme, also religiöse Sinnwelten, ihre Integration allein oder vor allem dadurch bewerkstelligen, dass sie für eine gleiche Ausrichtung ihrer jeweiligen Angehörigen sorgen - Stichwort >common beliefs se ist insofern unterkomplex, als sie das vielfältige Ineinander-Verstrebtsein von Sinnsphären unterschiedlicher interner (d.h. innersemantischer) und externer (d.h. den sozialen Alltag betreffender) Reichweite unberücksichtigt lässt. Nirgends, außer vielleicht in pädagogischen oder tota- 
litären Phantasien, sind atomistisch unverbundene Individuen in gleichartiger Weise auf einen gemeinsamen und als solchen identifizierbaren Sinn hin ausgerichtet. Selbst dort, wo so etwas vorübergehend oder in einem engen Radius gelingen mag, handelt es sich um einen schwachen Modus von sinnhafter Integration, zu dessen Aufrechterhaltung beträchtlicher Zwang erforderlich sein dürfte, weil keine interdependenten >Seitenstreben die Statik des Sinngebäudes stützen.

Auch für die Wirksamkeit von Glaubenssystemen müsste man stattdessen eine Art feldtheoretisches Modell entwickeln, das die Wechselwirkungen zwischen (immer partieller) Übereinstimmung und (immer partieller) Abweichung mit berücksichtigt. Spielen wir das in einem weiteren Gedankenexperiment durch: an dem hypothetischen Fall, dass irgendwo eine neue Glaubensrichtung entsteht und sich erfolgreich als dominantes Sinnsystem etabliert. Damit ist notwendig verbunden, dass sich eine Trägerschicht des neuen Glaubens ausbildet - teils in Fortsetzung, teils durch Auflösung vorheriger Elitenstrukturen; denn Dissidenten stammen ja meist aus dem Randbereich von Eliten. Die neue Religion bringt also eigene Legitimationsexperten hervor, die ein vorhandenes Bedürfnis nach Sinn und Zusammenhalt befriedigen oder ein nichtvorhandenes zu wecken versuchen. Soweit sie erfolgreich sind, können sie dem bisherigen religiösen Establishment Zustimmung entziehen. All das führt zu Reaktionsbildungen im gesamten sozialen Feld, die sich wiederum wie ein verzögerter Rückstoß auf das gesamte Spektrum von Symbiosen, Bündnissen, Koexistenzen, Rivalitäten bis hin zu offener Gegnerschaft auswirken.

Man muss sich dabei - gegen den üblichen Sprachgebrauch - in Erinnerung rufen, dass Konsens nicht nur verbindend und Differenz nicht nur trennend wirkt: Es gibt ja neben der sozialen auch so etwas wie eine symbolische Arbeitsteilung (oder besser ausgedrückt: eine Arbeitsteilung im Symbolischen), und Arbeitsteilung ist bekanntlich ein Modell für die verbindende Kraft von Differenzierung. Aber auch semantische AntiBildungen können paradoxerweise zur Integration beitragen, insofern sie unfreiwillig die Dominanz eines neuen Sinnkomplexes beglaubigen. Dissidenz mag auf Wandel abzielen, ist aber nicht gleichbedeutend mit Desintegration.

Wenn sich also ein neues Sinnsystem etabliert und dominant setzt, organisieren sich auch soziale Distinktionen, Funktionsteilungen, Dissonanzen neu. Alte Zwänge bzw. Abhängigkeiten lockern sich; die Dislozierung von symbolischem Kapital führt auch eine Umgewichtung anderer Kapitalien herbei; `Zulieferbetriebe` und Infrastruktureinrichtungen, sekundäre >Sinndienstleistungen< etc. organisieren sich um; dadurch entsteht lokaler >Sinnstress« (durch starke Sektenbildung, Exklusivität, Fanatisierung) mit liebsamen und unliebsamen Folgen; eine Gegenelite formiert sich, die aber in der Regel nach einiger Zeit in das herkömmliche Establishment einwandert; mit dem neuen Zentrum der Sinnwelt bilden sich auch neue be- wohnbare Peripherien, Freiräume, Übergangszonen etc. Das Ensemble all dieser Interdependenzen erzeugt ein labiles Gemisch von Integration und Variabilität, in dem auch Dissens bis zu einem gewissen Grad ausagiert werden kann. Denn alle Hegemonien schaffen sich früher oder später ein antigraves Kraftfeld, in dem sich Gegenkräfte, Gegenimpulse sammeln.

Die Grundgegebenheit von Glaubenssystemen ist also nicht Einsinnigkeit - einlinige Ausrichtung aller Gläubigen auf ein identifizierbares Glaubenssubstrat hin --, sondern >Streuung(. Soziale Streuung zieht semantische Streuung nach sich, und umgekehrt. Auch >Sinn< ist damit nicht einfach Medium von Integration, sondern in gleichem Maß von Auflösung und Zerstreuung. Er zieht sich sozusagen lokal zusammen, erreicht eine größere Verteilungsdichte rings um bestimmte Legitimationszentren, bricht sich mit anderen Sinnformen und dissipiert über das soziale Feld. Gesellschaften in ihrer Totalität (was immer das sein mag) sind nicht über Sinn integriert; das ist nur die interessierte Darstellung bestimmter Legitimationsexperten, die ihre spezialistische Sicht für die soziale Wirklichkeit überhaupt nehmen.

VI.

Worin besteht der Gewinn, so zu argumentieren - außer dass man ein vergleichsweise überschaubares Modell (Sinnprovinzen aggregieren sich und werden in einheitlichen Sinnwelten zusammengefasst, die uns vor dem Nichts oder dem Absurden abschirmen) durch ein unübersichtliches ersetzt? Ich denke, eine Kulturtheorie, die so verfährt, wird sich von bestimmten normativen, um nicht zu sagen metaphysischen Implikationen lösen. Sie wird Kultur nicht als eine Identität und Sinn stiftende bzw. verbürgende Instanz, sondern als offenen Raum ihrer differenziellen Konfigurationen und Verteilungen begreifen. Das rückt die Kulturtheorie weg von Traditionen der Theologie, Moral und Kulturphilosophie und näher an technische Betrachtungsweisen heran: an feldtheoretische Ansätze in anderen Wissenschaften, an die Kybernetik und an diejenigen sozialwissenschaftlichen Disziplinen, die sich mit Organisation, Distribution und insbesondere mit Infrastrukturproblemen befassen.

\section{Literatur}

Berger, Peter L./Luckmann, Thomas (2004). Die gesellschaftliche Konstruktion der Wirklichkeit. Eine Theorie der Wissenssoziologie. Frankfurt/Main: Fischer.

Giesen, Bernhard (2004). )Latenz und Ordnung. Eine konstruktivistische Skizze«. In: Ders./Osterhammel, Jürgen/Schlögl, Rudolf (Hg.): Die Wirklichkeit 
der Symbole. Grundlagen der Kommunikation in historischen und gegenwärtigen Gesellschaften. Konstanz: Universitätsverlag Konstanz. S. 73-100.

Hillmann, Karl Heinz (1994). »Art. >Sinn«《. In: Wörterbuch der Soziologie. Stuttgart: Kröner. S. 781-784.

Luhmann, Niklas (1988). Soziale Systeme. Grundriß einer allgemeinen Theorie. Frankfurt/Main: Suhrkamp.

Weber, Max (1956). Wirtschaft und Gesellschaft. Grundriss der verstehenden Soziologie. Tübingen: Mohr. 